\title{
Wild-type p53-modulated autophagy and autophagic fibroblast apoptosis inhibit hypertrophic scar formation
}

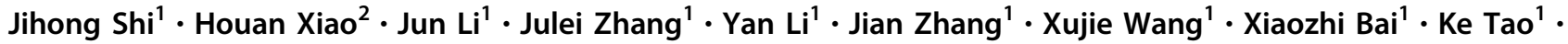 \\ Dahai $\mathrm{Hu}^{1} \cdot$ Hao Guan ${ }^{1}$
}

Received: 12 October 2017 / Revised: 12 April 2018 / Accepted: 24 May 2018 / Published online: 8 August 2018

(c) United States \& Canadian Academy of Pathology 2018

\begin{abstract}
Hypertrophic scarring is a serious fibrotic skin disease, and the abnormal activation of hypertrophic scar fibroblasts (HSFs) intensifies its pathogenesis. Our previous studies have demonstrated that the dysregulation of autophagy in HSFs is associated with fibrosis. However, knowledge regarding the regulation of HS fibrosis by p53-modulated autophagy is limited. Here, we investigated the effect of p53-modulated autophagy on HS fibrosis. The overexpression of wtp53 (Adp53) promoted autophagic capacity and inhibited collagen and $\alpha$-SMA expression in HSFs. In contrast, LC3 (AdLC3) overexpression did not suppress Col 1, Col 3, or $\alpha$-SMA expression, but LC3 (shLC3) knockdown downregulated collagen expression. Adp53-modulated autophagy altered Bcl-2 and Bcl-xL expression, but AdLC3 affected only Bcl-xL expression. Silencing Bcl-xL suppressed collagen expression, but autophagy was also inhibited. Flow cytometry showed that the silencing of Bcl-2 (sibcl-2), Bcl-xL (sibcl-xL), and Adp53 significantly increased apoptosis in the HSFs. Therefore, wtp53 inhibited fibrosis in the HSFs by modulating autophagic HSF apoptosis; moreover, the inhibition of autophagy by sibcl-xL had antifibrotic effects. In addition, treatment with Adp53, AdLC3, shLC3, sibcl-2, and sibcl-xL reduced scar formation in a rabbit ear scar model. These data confirm that wtp53-modulated autophagy and autophagic HSF apoptosis can serve as potential molecular targets for HS therapy.
\end{abstract}

\section{Introduction}

Hypertrophic scarring is a serious fibrotic skin disease characterized by over proliferation, dermal fibroblast

These authors contributed equally: Jihong Shi, Houan Xiao, Jun Li.

Electronic supplementary material The online version of this article (https://doi.org/10.1038/s41374-018-0099-3) contains supplementary material, which is available to authorized users.

Dahai $\mathrm{Hu}$

hudhai@fmmu.edu.cn

$\triangle$ Hao Guan

biojhshi@126.com

Jihong Shi

biojhshi@126.com

1 Department of Burns and Cutaneous Surgery, Xijing Hospital, Fourth Military Medical University, 127 West Chang-le Road, Xi'an, China

2 Burn Center of Xi' an Ninth Hospital Affiliated to the Medical College of Xi' an Jiaotong University, Xi' an, China activation, and excessive extracellular matrix (ECM) accumulation [1-8]. Previous studies investigating hypertrophic scar (HS) formation have focused on the imbalanced secretion of various cytokines, such as TGF- $\beta 1$ [9-11], which is suggested to stimulate the growth of granulation tissues and promote the transformation of fibroblasts into proliferative and more contractile myofibroblasts [12]. Myofibroblasts can be identified by their expression of alpha smooth muscle actin ( $\alpha$-SMA); in turn, myofibroblasts secrete abundant levels of collagen-based ECM proteins that cause scar formation and skin fibrosis. However, currently, there is no effective, therapeutically relevant target for HS treatment, primarily because its molecular mechanism is unclear.

Autophagy is a cellular response that sustains cellular metabolism during stress [13, 14]. However, prolonged autophagy activation can also result in the dysfunction of cellular organelles and even the self-destruction of cells $[15,16]$. Autophagy can also influence cell death and survival by regulating the apoptotic cascade (defined as type II programmed cell death). This process is physiologically essential for the maintenance of cellular functions, cell 
viability, differentiation and development in mammals, and serves as an adaptive mechanism that protects organisms from diverse pathological insults [15, 17-28]. Previous studies have shown that autophagy plays important roles in the pathogenesis of many human diseases and is associated with homeostasis in tissue structures and functions. Therefore, autophagy and autophagic apoptosis are associated with the maintenance, viability, differentiation, and survival of dermal fibroblasts during wound healing and lead to the formation and fibrosis of HSs [29].

p53 is a nuclear transcription factor that has been shown to play an important role in controlling cell proliferation and apoptosis [30, 31]. The dysregulation of dermal fibroblast proliferation and apoptosis is thought to be related to HS formation [32-34]. However, the relationship between HSs and p53 and the key molecules that regulate autophagy in HS fibroblasts (HSFs) are unclear. Our previous studies [24, 35] have shown that HS formation is related to HSF autophagy. Therefore, we sought to investigate the mechanism by which wild-type p53 (wtp53) modulates HSF autophagy and autophgic $\mathrm{HSF}$ apoptosis to regulate HS formation to identify a key molecular target for HS therapy.

\section{Materials and methods}

\section{Collection and processing of HS tissues}

HS and normal dermal skin (NS) tissues [6, 35] were collected from patients who underwent surgical excision at Xijing Hospital (Xi'an, China). Written consent was obtained from all participants before surgery. All protocols used in the study were approved by the Ethics Committee of Xijing Hospital, Affiliated to the Fourth Military Medical University of China. Each collected skin tissue sample was divided into the following two portions: one portion was preserved in $10 \%$ buffered formalin solution for immunostaining, and the other portion was used to isolate fibroblasts for culture.

\section{Immunostaining}

Immunostaining was performed as previously reported [6, 35]. In brief, skin tissues fixed in $10 \%$ buffered formalin were embedded in paraffin blocks and cut into 4- $\mu$ m-thick tissue sections. Then, the processed tissue sections were dewaxed and treated with $3 \%$ hydrogen peroxide for $15 \mathrm{~min}$, followed by blocking with goat serum for $30 \mathrm{~min}$, incubation at $4^{\circ} \mathrm{C}$ overnight with primary monoclonal antibodies (mAb) against LC3B (1:100, Cell Signaling) and Bcl-xL (1:100, Cell Signaling), and immunostaining using an SP-9000 Histostain ${ }^{\mathrm{TM}}$ Kit (Beijing Zhongshan Golden Bridge Biotechnology Co., China) according to the manufacturer's instructions. Briefly, tissue sections/cells on slides were incubated with a biotinylated secondary antibody, treated with streptavidin-biotin-horseradish peroxidase for signal amplification, and then stained with diaminobenzidine (DAB). Finally, tissue sections/cells on slides were counterstained with hematoxylin. Isotype-matched IgG was used as a negative control for each immunostaining procedure.

An immunofluorescence analysis was performed as previously reported $[6,35]$. In brief, the cells were grown on coverslips for $24-36 \mathrm{~h}$ until $70-80 \%$ confluent; then, the cells were fixed in $4 \%$ formaldehyde for $30 \mathrm{~min}$, washed with phosphate-buffered saline (PBS), permeabilized with $0.1 \%$ Triton-X100 for $10 \mathrm{~min}$ at room temperature, blocked with $1 \%$ bovine serum albumin (BSA), hybridized with a rabbit mAb specific for LC3B (1:100, Cell Signaling, CST) at room temperature for $1 \mathrm{~h}$, and incubated with a Cy3conjugated goat anti-mouse secondary antibody (1:100 dilution, Cwbio, China) at $37^{\circ} \mathrm{C}$ for $1 \mathrm{~h}$. Finally, the samples were stained with $4^{\prime}, 6^{\prime}$-diamidino-2-phenylindole (DAPI, Sigma).

\section{Transmission electron microscopy (TEM)}

Ultrathin HSFs sections were processed using conventional methods [35]. The samples were fixed with $2.5 \%$ glutaraldehyde in PBS buffer, embedded in agar, rinsed with Sorensen's buffer, post-fixed in osmium tetroxide $1 \%$ in Sorensen's buffer, dehydrated in ethanol, and embedded in EPON resin. Ultrathin sections were cut and stained with uranyl acetate and lead citrate and examined under a JEM-123 transmission electron microscope (JEOL, Japan) at $80 \mathrm{kV}$.

\section{Cell culture and treatment}

Cell culture was performed as previously described [6, 35]. Briefly, the fibroblasts were extracted from minced HS tissues by incubation in HBSS (Hank's balanced salt solution) with collagenase type I $(0.1 \mathrm{mg} / \mathrm{mL}$, Sigma $)$ at $37^{\circ} \mathrm{C}$ for 2.5 h. The extracted HSFs were collected and cultured at $37^{\circ} \mathrm{C}$ (in a $5 \% \mathrm{CO}_{2}$-humidified incubator) in Dulbecco's Modified Eagle's Medium (DMEM, Gibco) supplemented with $10 \%$ fetal calf serum (FCS, Gibco), $100 \mathrm{U} / \mathrm{mL}$ penicillin, and 100 $\mathrm{U} / \mathrm{mL}$ streptomycin (HyClone). All experiments were performed using cells at passage 3-5. Biochemical analyses were conducted using HSFs at $70-80 \%$ confluence after incubation for $12-16 \mathrm{~h}$ in serum-free medium.

\section{Infection with overexpression (Ad-) and knockdown (sh-) adenovirus vectors and transfection with siRNA}

The recombinant adenovirus vectors for wtp53 (Adp53) and LC3B (AdLC3) and the wtp53 (shp53, target sequence: gactccagtggtaatctac) and LC3B (shLC3, target sequence: cgcttacagctcaatgctaat) knockdown and the control adenovirus vector containing non-targeting (AdNT) shRNA 
Table 1 Sequences of siRNA

\begin{tabular}{lll}
\hline Type of gene & Forward $\left(5^{\prime} \rightarrow 3^{\prime}\right)$ & Reverse $\left(5^{\prime} \rightarrow 3^{\prime}\right)$ \\
\hline Bcl-2 & CCCUGUGGAUGACUGAGUATT & UACUCAGUCAUCCACAGGGTT \\
Bcl-xL & AUUGGUGAGUCGGAAUGGCATT & UGCGAUCCGACUCACCAAUTT \\
Negative control & UUCUCCGAACGUGUCACGUTT & ACGUGACACGUUCGGAGAATT \\
\hline
\end{tabular}

Table 2 Primers of qRT-PCR

\begin{tabular}{lll}
\hline Type of gene & Forward $\left(5^{\prime} \rightarrow 3^{\prime}\right)$ & Reverse $\left(5^{\prime} \rightarrow 3^{\prime}\right)$ \\
\hline Col 1 & GAGGGCAACAGCAGGTTCACTTA & TCAGCACCA CCGATGTCCA \\
Col 3 & CCACGGAAACACTGGTGGAC & GCCAGCTGCACATCAAGGAC \\
$\alpha$-SMA & GACAATGGCTCTGGGCTCTGTAA & TGTGCTTCGTCACCCACGTA \\
GAPDH & GCACCGTCAAGCTGAGAAC & TGGTGAAGACGCCAGTGGA \\
\hline
\end{tabular}

(shNT) were purchased from Genomeditech (Shanghai, China). All vectors were labeled with GFP, which served as a detection marker. HSFs grown to $50-70 \%$ confluence were incubated for $12 \mathrm{~h}$ in serum-free medium and infected with the vectors for $48 \mathrm{~h}$ to generate stable cells. For the siRNA (GenePharma, Shanghai, China) transfection, the HSFs were seeded in complete media in 6-well dishes on the day prior to the experiment. For all assays, the concentration of siRNAs during transfection was $10 \mathrm{nM}$. All transfections were performed in a mixture of Opti-MEM and complete media without antibiotics as previously described [36, 37]. The siRNA duplexes for Bcl-xL, Bcl-2, and the scramble control sequences are listed in Table 1. The transfection incubation time for the siRNA/Lipofectamine RNAiMAX reagent complexes (Life Technologies) was $24 \mathrm{~h}$. The total incubation time before cell lysis and protein isolation was $48 \mathrm{~h}$. The efficiency of the transfection was performing by a western blot analysis.

\section{qRT-PCR}

qRT-PCR was performed as previously reported [6, 35]. In brief, the total RNA was extracted from cultured cells using an RNA isolation kit (Takara, Japan). The purity of the RNA was calculated as follows: A260/A280 (1.9-2.0). The human primer pairs used for the gene amplification from the cDNA template are listed in Table 2. The relative expression of the target gene transcripts is expressed as the mean abundance in three independent reactions. The expression levels were normalized to those of GAPDH.

\section{Western blot analysis}

Cultured HSFs were harvested, washed with PBS, and resuspended in RIPA cell lysis solution (Beyotime, China) supplemented with $200 \mu \mathrm{g} / \mathrm{mL}$ phenylmethylsulfonyl fluoride (PMSF, Boster, China), phosphatase inhibitor cocktail (Sigma), and protease inhibitor cocktail (Sigma). The protein concentration of the cell lysates was determined using a BCA assay (Pierce).

Western blotting was performed as previously described $[6,35]$. Briefly, cell lysates containing equal amounts of protein were separated on $8 \%$ (for $\mathrm{Col} 1$ and $\mathrm{Col} 3$ ) or $14 \%$ (for LC3) SDS-PAGE gels and transferred onto polyvinylidene fluoride (PVDF, Millipore) membranes at $100 \mathrm{~V}$ for $30 \mathrm{~min}$ (LC3, Bcl-2, and $\mathrm{Bcl}-\mathrm{xL}$ ) or $100 \mathrm{~min}$ (Col 1, Col 3, and Beclin1). Then, the membranes were blocked with 5\% non-fat milk in TBST (Tris-buffered saline $/ 0.5 \%$ Tween-20) at room temperature for $3 \mathrm{~h}$, followed by incubation at $4{ }^{\circ} \mathrm{C}$ overnight with rabbit/mouse mAbs specific for Bcl-2 (Cell Signaling, 15071), Bcl-xL (Cell Signaling, 2764), Col 1 (Abcam, ab96723), Col 3 (Abcam, ab7778), alpha-actin (Epitomics), LC3B (Cell Signaling, 3868), Beclin1 (Cell Signaling, 3495), p62 (Cell Signaling), and wtp53 (Millipore, 2397843; Abcam, ab16776, ab7757). Finally, the membranes were washed and incubated with HRP-conjugated secondary antibodies (1:3000, Bioss, China). The immunoreactive protein bands were detected using ECL reagents (Millipore). The signal intensity of each protein band was quantified by scanning the membrane using an image analyzer (Alpha Innotech, USA). Then, the membrane was stripped of antibodies and re-probed with a rabbit $\mathrm{mAb}$ against $\beta$-actin (1:2000 dilution, Cell Signaling) as an internal loading control.

\section{Rabbit ear scarring model and treatment}

We used a previously described rabbit ear scarring model in this study [36, 37]. New Zealand white rabbits weighing 2.0-2.5 kg (permit number 20160203) were purchased from the Experimental Animal Center of the Fourth Military Medical University and maintained in separate cages. The animal experiments were approved by the Experimental Animal Committee of the Fourth Military Medical University. The animals were anesthetized using an intravenous administration of sodium pentobarbital $(30 \mathrm{mg} / \mathrm{kg})$. In a sterile environment, four full-thickness wounds down to the 
cartilage were created on each ear. Four round, 10-mm diameter wounds were randomly created on the ear. Thus, each rabbit had eight wounds. In each wound, the epidermis, dermis, and perichondrium were completely removed. Then, the wounds were covered with sterile gauze for 1 day. The rabbits were returned to their cages upon recovery from anesthesia. After scar formation, which occurred 28 days post operatively, the scars were randomly allocated to the following ten groups (six scars per group): one PBS group, two control groups (AdNT and shNT), one siRNA control groups (siNT), and six treatment groups (Adp53, shp53, AdLC3, shLC3, sibcl-2, and sibcl-xL). Then, $5 \mathrm{nM}$ siRNAs or $1 \times 10^{6} \mathrm{CFU}$ in $100 \mu \mathrm{l}$ PBS were injected intra-dermally into the scars. The ears were observed every 7 days. The rabbits were killed on the 49th day. The harvested specimens were analyzed using H\&E and Masson trichrome staining. We performed light microscopy to examine the degree of scar hyperplasia, which was represented by the scar elevation index (SEI) or the ratio of the height of the scar tissue to the height of the normal tissue below the HS. A ratio of 1 indicates no difference between the height of the wound area and the height of the unwounded skin (Fig. 7b).

\section{Flow cytometry}

FITC/PE Annexin V Apoptosis Detection Kits (BD Pharmingen ${ }^{\mathrm{TM}}$ ) were used to measure HSF apoptosis after transfection (siRNAs) or infection (recombinant adenovirus) [38-40]. Briefly, the HSFs were treated with medium alone, siRNA specific for Bcl-xL, negative control siRNA, or Lipofectamine RNAiMAX Reagent (Life Technologies) only for $48 \mathrm{~h}$. The HSFs were washed with PBS, resuspended in $100 \mu$ l binding buffer and stained with $5 \mu$ FITCAnnexin $\mathrm{V}$ and propidium iodide (PI) for $15 \mathrm{~min}$ in the dark; then, $300 \mu$ of binding buffer were added. For the recombinant adenovirus, the HSFs were collected after infection for $48 \mathrm{~h}$. Analyses were performed using a flow cytometer (BD FACSAria ${ }^{\mathrm{TM}}$ III system; BD Pharmingen) according to the manufacturer's instructions. The HSFs in the FITC/PEpositive fraction were considered apoptotic cells.

\section{Results}

\section{Collagen-based ECM and autophagic capacity increase in HSs and HSFs}

The histological observations were performed using HS and normal skin (NS) tissues. Both H\&E and Masson's trichrome staining showed numerous fibroblasts in the dermis of the HS tissue (Fig. 1a-c, g-i), whereas a lower density of fibroblasts was observed in the NS tissue (Fig. $1 \mathrm{~d}-\mathrm{f}, \mathrm{j}-\mathrm{l}$ ). The excessive accumulation of collagens and keratinization in the epidermis was representative HS lesion features (Fig. 1g-i). While the collagen fiber bundles appeared to be organized as a network in the NS tissue (Fig. 1j-l), they appeared as disordered bundles in the HS tissue (Fig. 1a-c, 1g-i). To investigate the differential expression of fibrosis-related genes in the HS tissue and primary HSFs, the transcription levels of Col 1, Col 3, and $\alpha$-SMA were analyzed by performing qRT-PCR. The Col 1, Col 3, and $\alpha$-SMA transcription levels in the HS tissues were significantly higher than those in the corresponding NS tissues (Fig. 1m). The transcription levels in the HSFs were also higher than those in the NSFs (Fig. 1m). These results showed that the HSs constitute significant fibrotic dermal skin tissue characterized by disordered collagen-based ECM protein deposition.

Based on the ultra-structure analysis, the number of autophagosomes in the HS/HSFs was significantly higher than that in the NS/NSFs (Fig. S1a, b). The immunocytochemistry data showed that although LC3 was localized in both NS and HS, its expression level was significantly higher in HS (Fig. S1c). The immunofluorescence results showed that LC3 was localized in the cell cytoplasm in the $\mathrm{NSFs} / \mathrm{HSFs}$, and its expression in the HSFs was significantly higher than that in the NSFs (Fig. S1d). These results showed that the autophagic capacity in the HS/HSFs was higher than that in the NS/NSFs.

\section{Wtp53 represses the expression of $\mathrm{Col} 1, \mathrm{Col} 3$, and a-SMA in HSFs}

To verify the antifibrosis role of wtp53, adenoviruses for the overexpression of wtp53 (Adp53) and knocking down of wtp53 (shp53) and their negative controls (AdNT and shNT) were generated and used to infect cultured HSFs. The qRT-PCR analyses showed that Adp53 significantly decreased the transcriptional levels of $\alpha$-SMA (Fig. 2a), Col 1 (Fig. 2b), and Col 3 (Fig. 2c) in the HSFs. Shp53 had no effect on the levels of $\alpha$-SMA (Fig. 2a), Col 1 (Fig. 2b) and Col 3 (Fig. 2c) in the HSFs. Similarly, the western blot analyses showed that Adp53 significantly downregulated the expression of $\alpha$-SMA (Fig. 2d, e), Col 1 (Fig. 2d, f), and Col 3 (Fig. 2d, g) in the HSFs. Shp53 had no effects on the expression of $\alpha$-SMA (Fig. 2d, e), Col 1 (Fig. 2d, f), and Col 3 (Fig. 2d, g) in the HSFs. These results indicated that wtp53 inhibited the expression of $\mathrm{Col} 1, \mathrm{Col} 3$, and $\alpha$-SMA in the HSFs.

\section{LC3 knockdown inhibits the expression of Col 1 and Col 3 and changes the architecture arrangement of a-SMA in HSFs}

To verify the antifibrotic role of LC3, adenoviruses for the overexpression of LC3 (AdLC3) and knockdown of LC3 

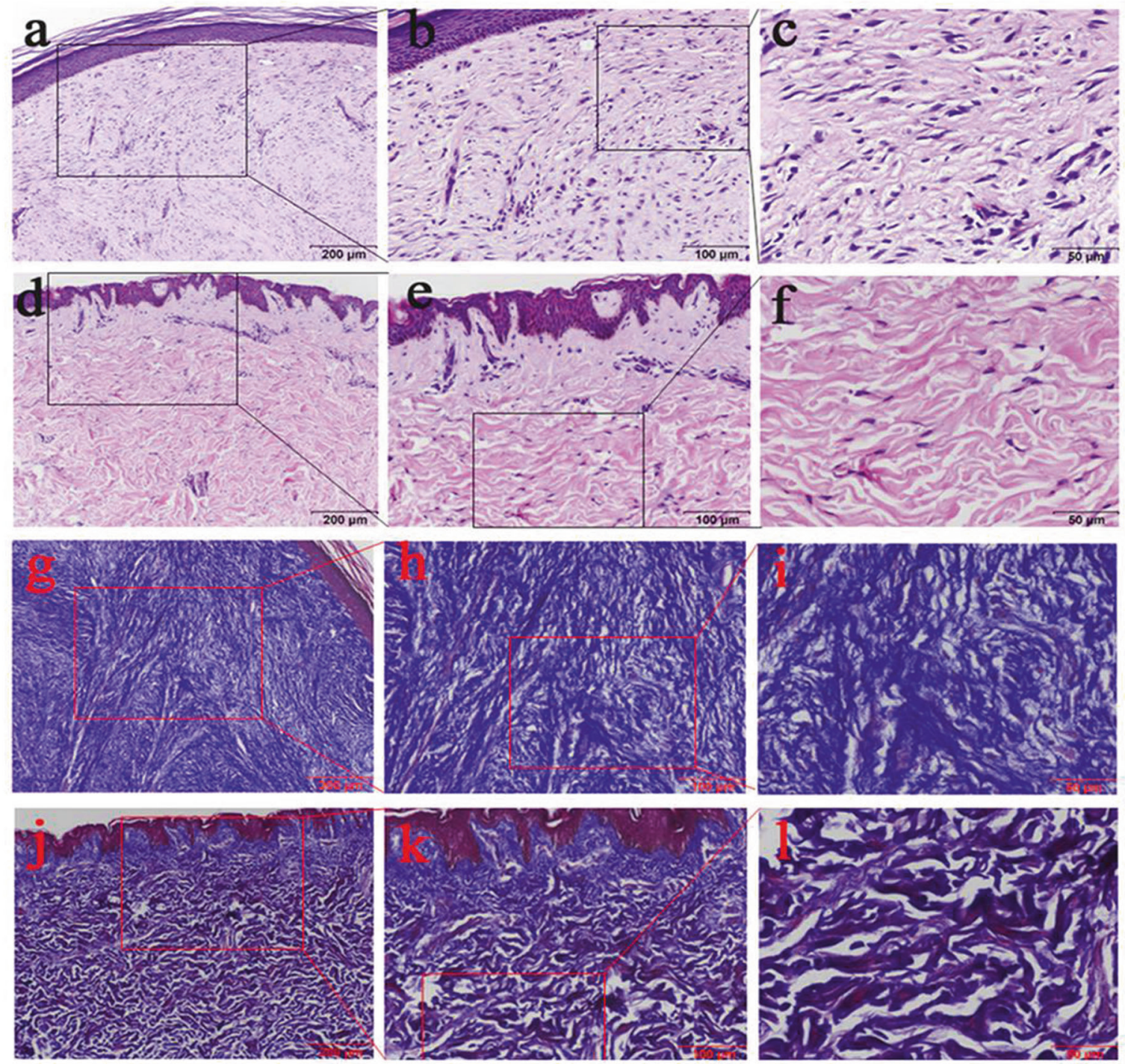

In

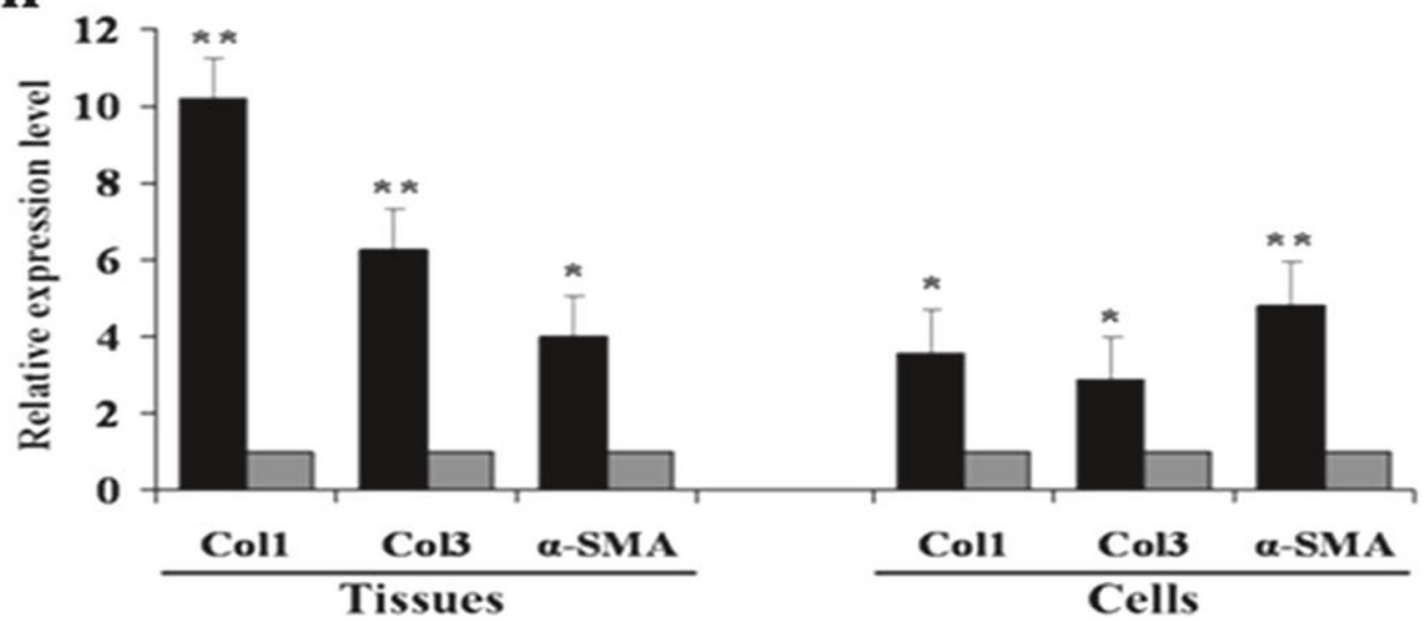

Fig. 1 Collagen-based ECM is elevated in HSs and HSFs. a-c H\&E staining of HSs. d-f H\&E staining of NS. g-i Masson's trichrome staining of HSs. $\mathbf{j}-\mathbf{l}$ Masson's trichrome staining of NS. Scale bars, 200,100 , and $50 \mu \mathrm{m}$. $\mathbf{m}$ The differential transcription levels of Col 1 , $\mathrm{Col} 3$, and $\alpha$-SMA were analyzed in HSs and primary HSFs by qRT-
PCR. The data are expressed as the mean \pm SEM; $n=3, * p<0.05$, $*^{* *} p<0.01$. These results show that HSs are significant fibrotic dermal skin tissues characterized by a dysregulation of collagen-based ECM proteins deposition 

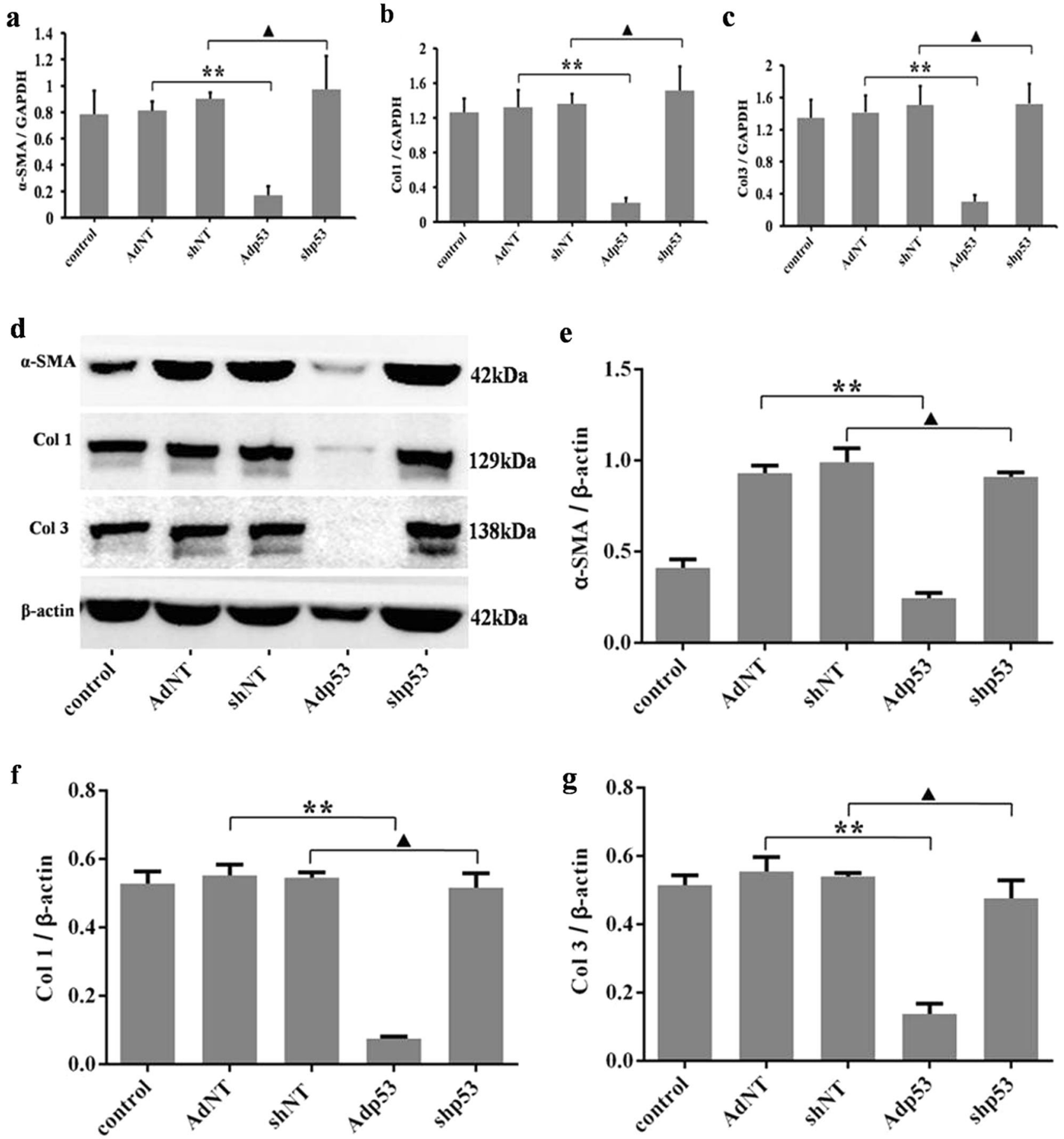

Fig. 2 Wtp53 overexpression inhibits the expression of collagen matrix proteins in HSFs. HSFs that were $50-70 \%$ confluent were infected with Adp53, shp53, and their negative control adenovirus vectors and cultured in DMEM for 36-48 $\mathrm{h}$; then, the mRNA and protein expression levels were detected by qRT-PCR and western blotting. a, b, $\mathbf{c}$ the mRNA expression levels of $\alpha$-SMA, Col 1 and Col 3.

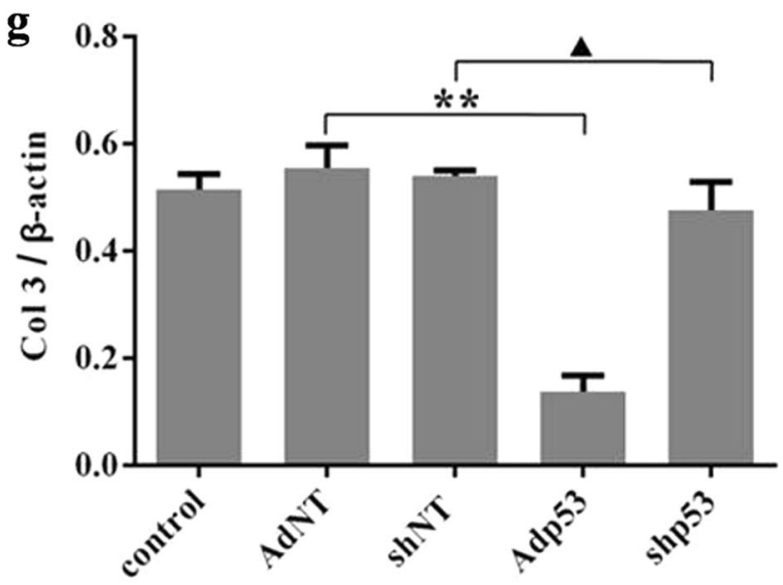

d, e $\alpha$-SMA protein expression and changes in the $\alpha$-SMA $/ \beta$-actin ratio. d, f Col 1 protein expression and changes in the Col $1 / \beta$-actin ratio. d, $\mathbf{g} \mathrm{Col} 3$ protein expression and changes in the $\mathrm{Col} 3 / \beta$-actin ratio. The data are expressed as the mean \pm SEM; $n=3, \boldsymbol{\Delta}_{p}>0.05$, $* p<0.05, * * p<0.01$ compared with the negative control

(shLC3) and their negative controls (AdNT and shNT) were used to infect HSFs. The western blot analyses showed that shLC3 significantly downregulated the

expression of Col 1 (Fig. 3a, c) and Col 3 (Fig. 3a, d) but did not affect the expression of $\alpha$-SMA in the HSFs (Fig. 3a, b). AdLC3 did not suppress the expression of Col 

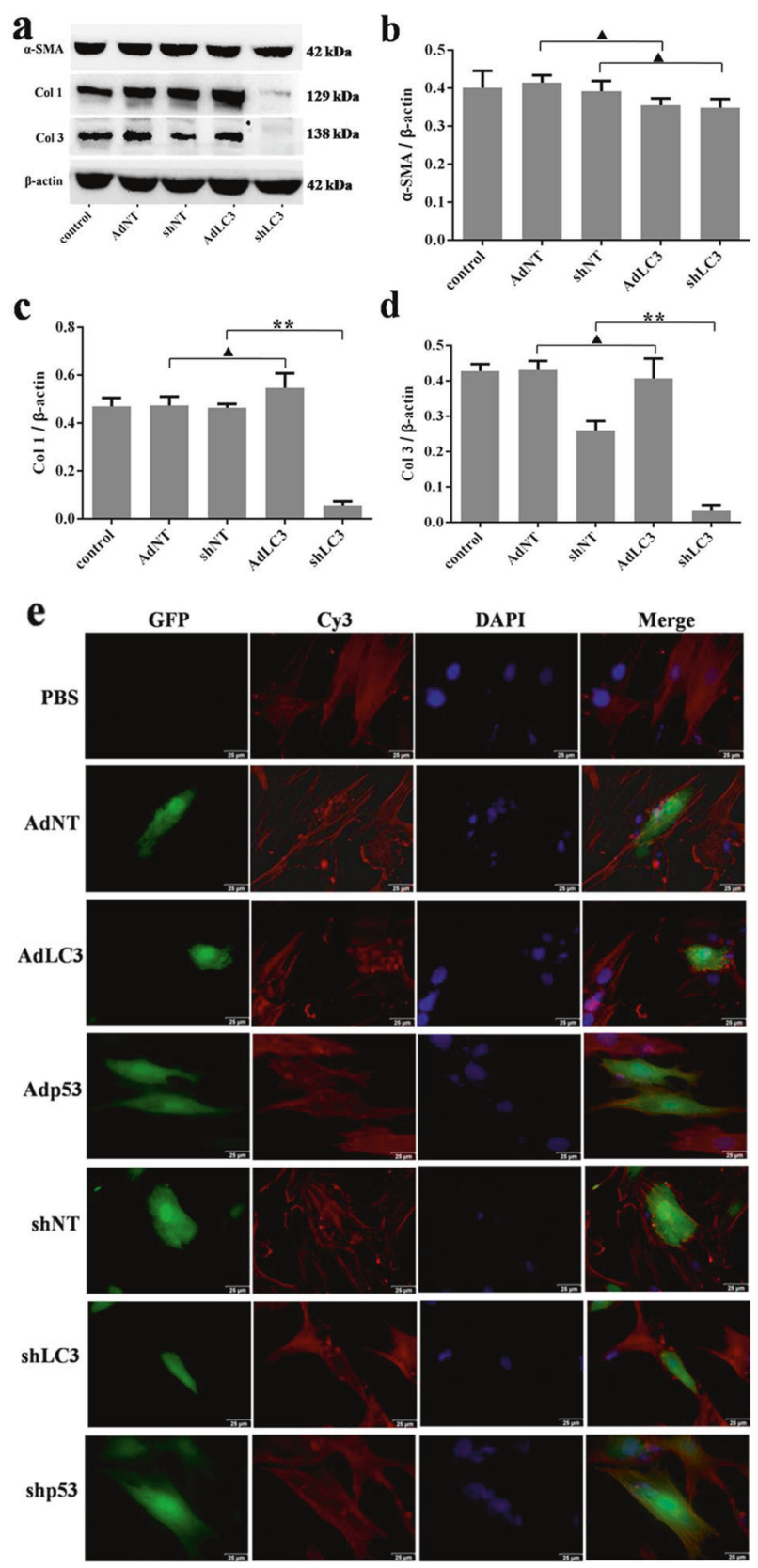

Fig. 3 Knocking down LC3 inhibits the expression of collagen matrix proteins and affects the architecture arrangement of $\alpha$-SMA in HSFs. HSFs were infected with AdLC3, shLC3, and their negative controls and cultured in DMEM for $36-48 \mathrm{~h}$; then, the protein expression levels were detected by western blotting. a, b $\alpha$-SMA protein expression and changes in the $\alpha$-SMA $/ \beta$-actin ratio. a, c Col 1 protein expression and changes in the Col $1 / \beta$-actin ratio. a, d Col 3 protein expression and changes in the $\mathrm{Col} 3 / \beta$-actin ratio. The data are expressed as the mean \pm SEM; $n=3, \boldsymbol{\Delta}_{p}>0.05, * p<0.05$, ${ }^{* *} p<0.01$ compared with the negative control. e The architecture arrangement of $\alpha$-SMA in the infected HSFs was analyzed by immunofluorescence. Scale bars, $50 \mu \mathrm{m}$

1 (Fig. 3a, c), Col 3 (Fig. 3a, d), or $\alpha$-SMA (Fig. 3a, d) in the HSFs. These results implied that shLC3 inhibited the expression of Col 1 and $\mathrm{Col} 3$ in the HSFs.
To further explore the effect of LC3 on myofibroblasts, we used an immunostaining method and observed $\alpha$-SMA in HSFs. Our results showed that $\alpha$-SMA displayed fiber filaments similar to those observed in the control groups (AdNT and shNT, Fig. 3e), but in the shLC3 and AdLC3 groups, the architecture arrangement of $\alpha$-SMA appeared as a tadpole and rod (Fig. 3e). These results indicated that shLC3 not only inhibited the expression of Col 1 and Col 3 (Fig. 3a, c, d) in the HSFs, but also changed the architecture arrangement of $\alpha$-SMA, whereas AdLC 3 changed only the architecture arrangement (Fig. 3e).

\section{Wtp53 promotes autophagy and downregulates Bcl-} 2 and $\mathrm{BCl}-\mathrm{xL}$ in HSFs

Wtp53 has an ability to cause cell senescence and apoptosis [29-31]. Recently, wtp53 has been shown to control autophagy in an ambiguous fashion [41, 42]. The western blot analyses showed that wtp53 increased the LC3-II/LC3-I ratio (Fig. 4a, b) and upregulated the expression of beclin1 (Fig. 4a, c), but it had no effect on the expression of p62 (Fig. 4a, d); shp53 exhibited the opposite effects (Fig. 4a-d). These results indicated that wtp53 significantly promoted the autophagic capacity in the HSFs.

Because the above results indicated that both Adp53 and shLC3 inhibited the expression of Col 1 and $\mathrm{Col} 3$ in the HSFs, we aimed to identify the molecule regulated by Adp53 and shLC3. We tested the Bcl-2 family molecules. Expectedly, the western blot analyses showed that Adp53 significantly inhibited the expression of Bcl-xL (Fig. 4a, e) and Bcl-2 (Fig. 4a, f), while shp53 had negative effects (Fig. 4a, e, f). In addition, AdLC3 increased the LC3-II/LC3-I ratio (Fig. 4g, h) and downregulated the expression of p62 (Fig. 4g, j) in AdLC3infected HSFs, but shLC3 had a negative effect or no effect (Fig. 4g-j). Moreover, AdLC3 significantly inhibited the expression of only Bcl-xL (Fig. 4g, k), not Bcl-2 (Fig. 4g, 1), and shLC3 did not inhibit Bcl-xL (Fig. 4g, l) or Bcl-2 (Fig. 4g, 1).

These results demonstrated that wtp53 promoted autophagy and inhibited Bcl-xL and Bcl-2, whereas AdLC3induced autophagy also promoted autophagy and inhibited $\mathrm{Bcl}-\mathrm{xL}$ expression. Therefore, $\mathrm{Bcl}-\mathrm{xL}$ may be an important factor that regulates or is influenced by autophagy. However, the effect of autophagy on collagen expression is obscure because wtp53-induced autophagy inhibited Col 1 and $\mathrm{Col} 3$, but AdLC3-induced autophagy had no effect; moreover, shLC3 inhibited collagen expression. In addition, the changes in Bcl-xL were not consistent with the changes in collagen expression. Thus, the roles of autophagy and $\mathrm{Bcl}-\mathrm{xL}$ in the expression of collagens must be further investigated. 
$\mathbf{a}$

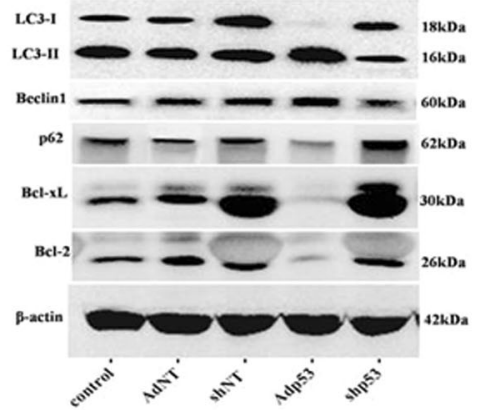

d

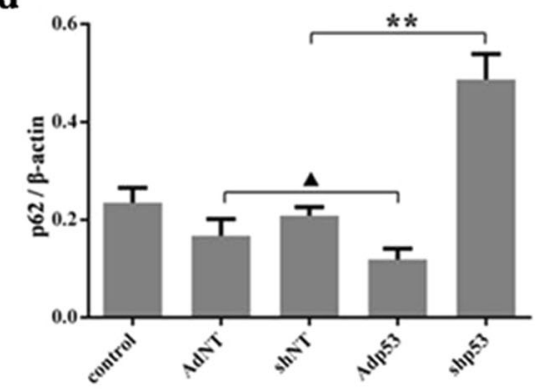

g

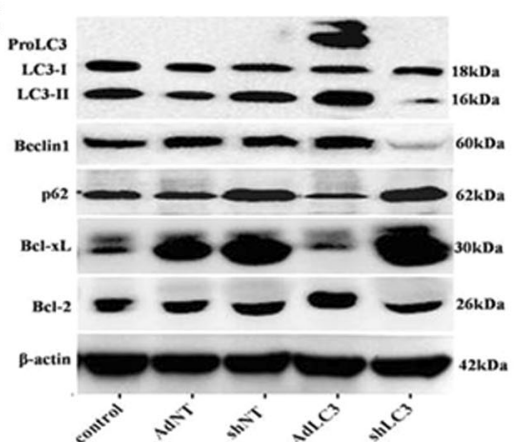

j

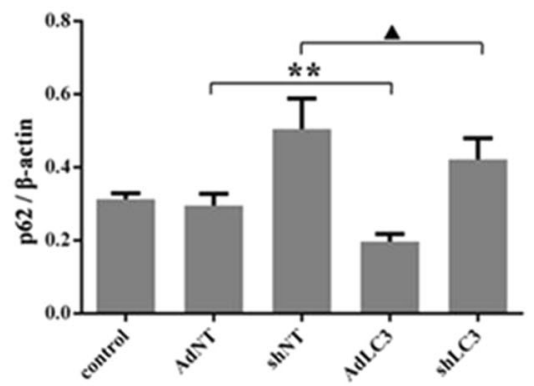

b

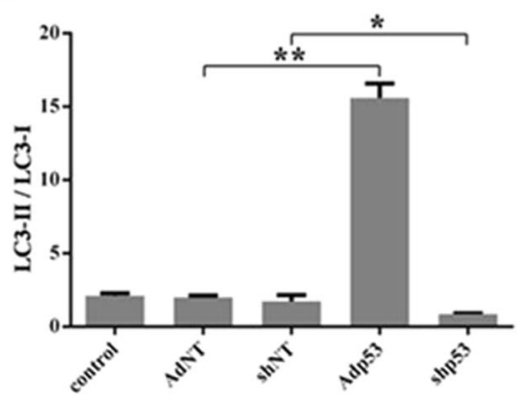

e

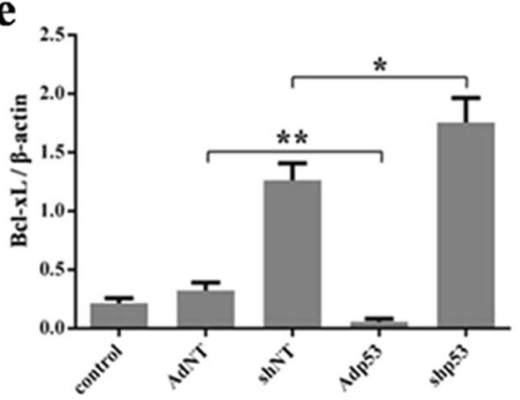

h

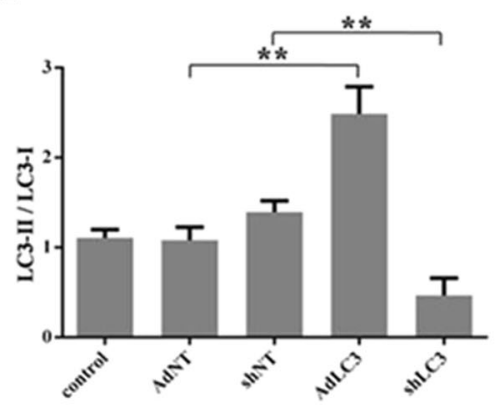

$\mathbf{k}$

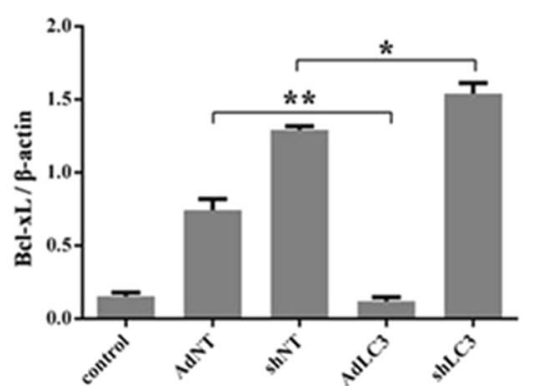

$\mathbf{c}$

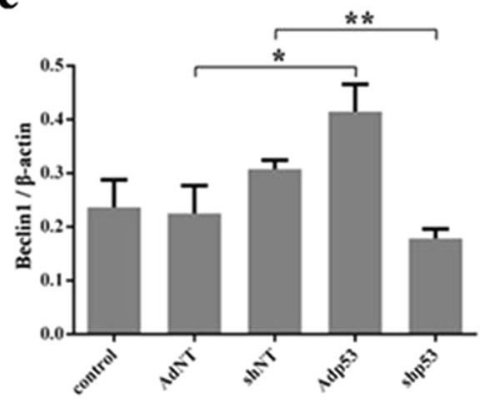

f

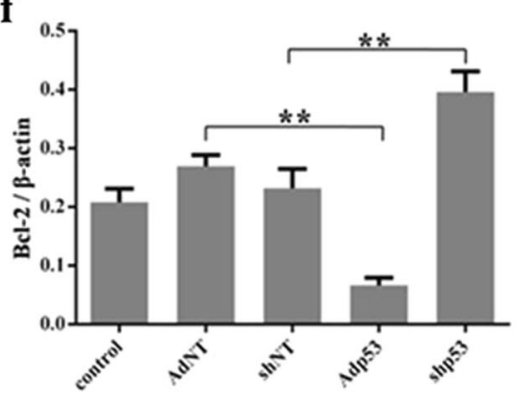

i

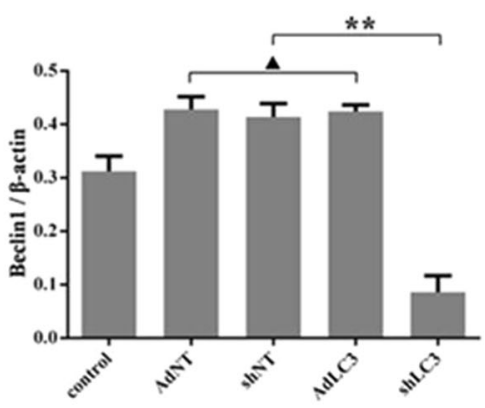

l

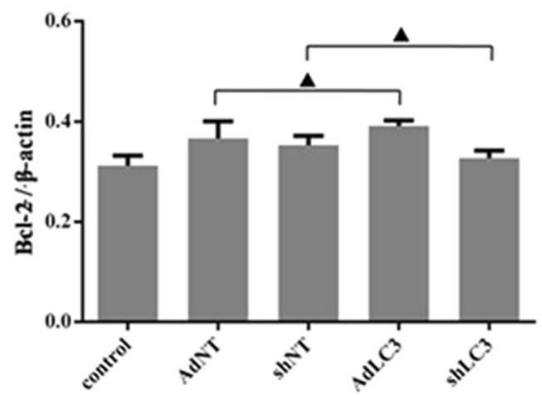

Fig. 4 Effects of wtp53 and LC3 on autophagic capacity and Bcl-xL and Bcl-2 expression in HSFs. HSFs that were 50-70\% confluent were infected with Adp53, shp53, and their negative control adenovirus vectors and cultured in DMEM for $36-48 \mathrm{~h}$; then, the HSFs were analyzed by western blotting. $\mathbf{a}, \mathbf{b}, \mathbf{g}, \mathbf{h}$ Changes in the ratio of LC3-II/LC3-L in the infected HSFs. a, c, g, i Autophagy protein

\section{Silencing Bcl-xL inhibits autophagy and the expression of collagens}

To understand the exact role of Bcl-xL in the regulating of the expression of ECM proteins in HSFs, sibcl-xL and
Beclin1 expression and changes in the Beclin $1 / \beta$-actin ratio. $\mathbf{a}, \mathbf{d}, \mathbf{g}, \mathbf{j}$ p62 expression and changes in the p62/ $\beta$-actin. a, e,g , $\mathbf{k ~ B c l - x L}$ protein expression and changes in the $\mathrm{Bcl}-\mathrm{xL} / \beta$-actin ratio. a, f, $\mathbf{g}, \mathbf{l} \mathrm{Bcl}-2$ protein expression and changes in the $\mathrm{Bcl}-2 / \beta$-actin ratio. The data are representative of three experiments. $\mathrm{n}=3, \boldsymbol{\Delta}_{\mathrm{p}}>0.05,{ }_{\mathrm{p}}<0.05$, $* * \mathrm{p}<0.01$ compared with the negative control group

sibcl-2 were transfected into HSFs (Fig. S2). The qRTPCR analyses showed that sibcl-xL, but not sibcl-2 (Fig. 5a, b), suppressed the transcription levels of Col 1 (Fig. 5a) and Col 3 (Fig. 5b) in the HSFs. The western blotting showed that sibcl-xL, but not sibcl-2 (Fig. 5d, f, 
Fig. 5 Silencing Bcl-xL inhibits autophagy and the expression of collagens. HSFs

were transfected with

siRNAs and cultured for 24 and $48 \mathrm{~h}$. a, b, c The mRNA levels of Col 1, Col 3, and $\alpha$-SMA were analyzed by qRT-PCR. d, e $\alpha$-SMA protein expression and changes. d, f Col 1 protein expression and changes. d, $\mathbf{g ~ C o l}$ 3 protein expression and changes. h, i Changes in the ratio of LC3-II/LC3-I in the HSFs. h, j Autophagy protein Beclin 1 expression and changes. $\mathbf{h}, \mathbf{k}$ p62 protein expression and changes. The data are representative of three experiments. $n=3, \boldsymbol{\Delta}_{p}>0.05$, $* p<0.05, * * p<0.01$ compared with the negative control group

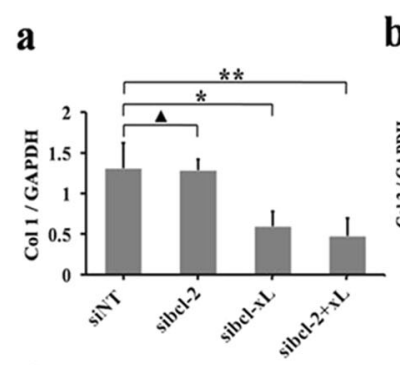

d

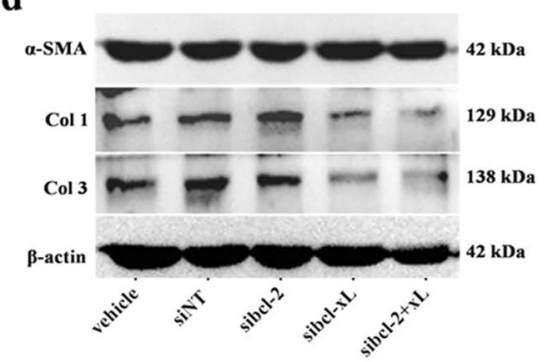

f

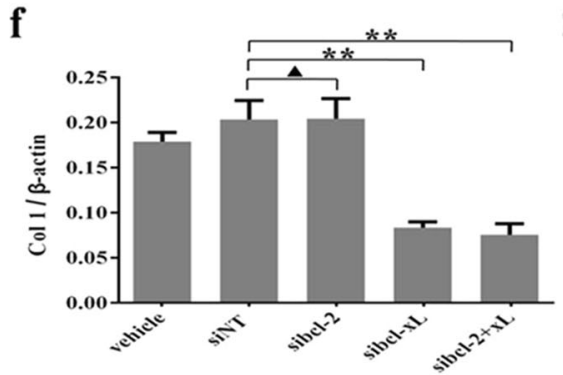

$\mathbf{h}$

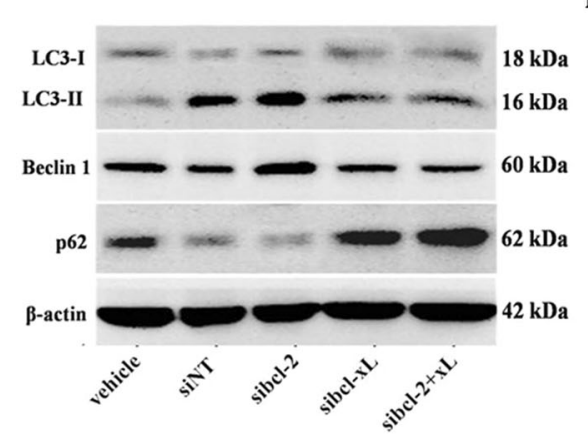

j

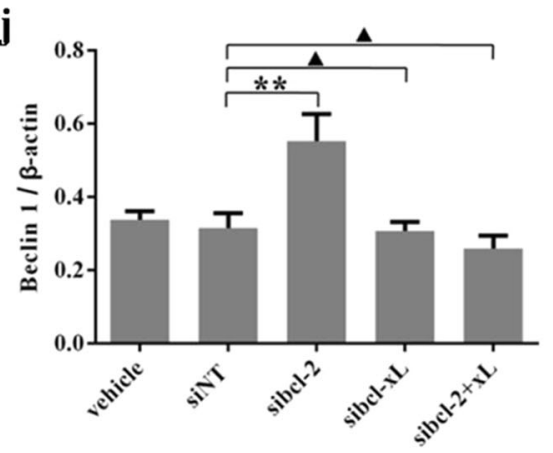

e b ${ }^{* * *}$ C
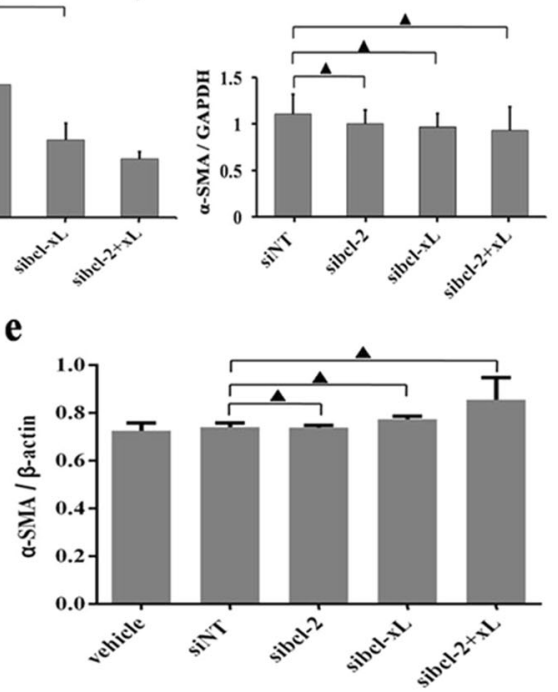

g

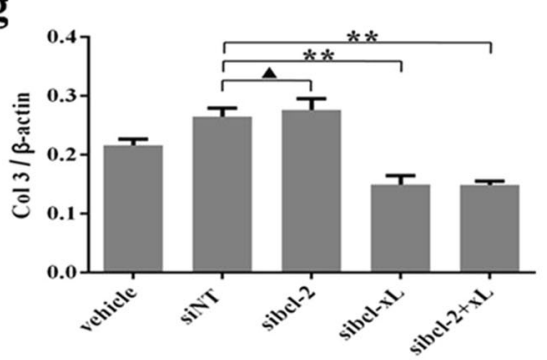

i

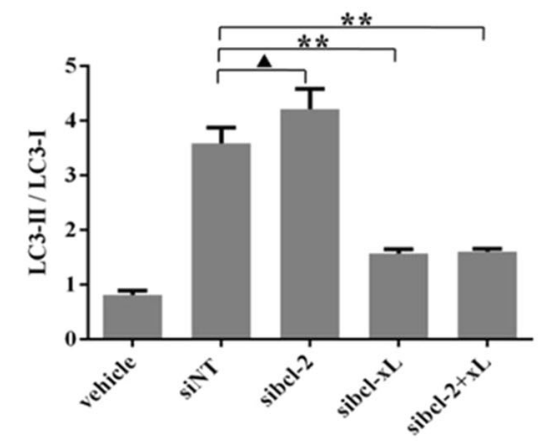

$\mathbf{k}$

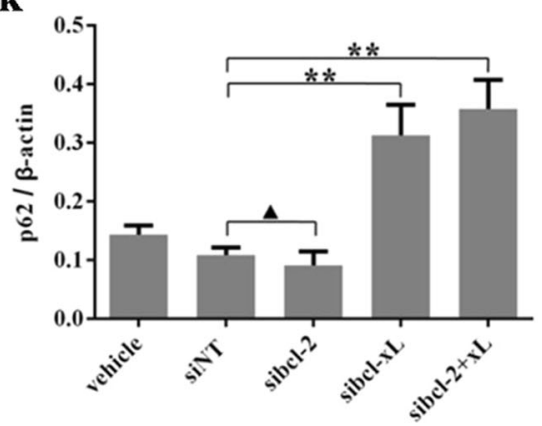

g), remarkably inhibited the expression of Col 1 (Fig. 5d, f) and $\mathrm{Col} 3$ (Fig. 5d, g) in the HSFs. Neither sibcl-xL nor sibcl-2 altered the transcription (Fig. 5c) or expression of
$\alpha$-SMA (Fig. 5d, e). These results indicated that Bcl-xL is a key signaling molecule involved in collagen metabolism in HSFs. 
To further determine the effect of Bcl-2 and Bcl-xL on HSF autophagy in fibroblasts, siRNAs (Fig. S2a-c) were used to transfect HSFs. The western blot analyses showed that sibcl-xL downregulated the expression of LC3 (Fig. 5h, i) and Beclin (Fig. 5h, j) and upregulated the expression of p62 (Fig. 5h, k) in the HSFs. These results indicated that sibcl-xL inhibited autophagy in the HSFs. As shown above, the downregulation of Bcl-xL induced by AdLC 3 and wtp53 was accompanied by increased autophagy, but sibcl$\mathrm{xL}$ was shown to decrease autophagy, which appears contradictory. Thus, we hypothesized that the downregulation of Bcl-xL by sibcl-xL inhibited autophagy, indicating that Bcl-xL plays a key role in autophagy. In contrast, the decreased Bcl-xL levels after the induction of autophagy by AdLC 3 and wtp53 could be a negative feedback of autophagy. However, the relationship between collagen and autophagy remains unclear.

\section{Autophagy and autophagic apoptosis regulate the expression of collagen}

p53 and the Bcl-2 family members have been reported to play key roles in apoptosis [43-45]. Thus, we investigated whether the changed collagen expression was related to apoptosis in the HSFs. Flow-based Annexin V assays were performed to determine the effects of Adp53, shLC3, sibcl2, and sibcl-xL on HSF apoptosis. As shown in Fig. 6a-c, sibcl-xL promoted the HSF apoptosis levels $48 \mathrm{~h}$ after transfection, and the apoptosis rates after the transfection with the Lipofectamine RNAiMAX Reagent alone, negative sequence (siNT), sibcl-xL, sibcl-2, and sibcl-xL+sibcl-2 (sibcl-2+xL) were $7.22 \pm 0.62 \%$ (Fig. 6a), $8.12 \pm 0.32 \%$ (Fig. 6b), $12.60 \pm 0.42 \%$ (Fig. 6c), $14.71 \pm 0.36 \%$ (Fig. 6d), and $17.59 \pm 0.29 \%$ (Fig. 6e), respectively. Specifically, the apoptosis rates in the HSFs transfected with sibcl-xL and sibcl-2, either alone or in combination, were significantly higher than those in the control groups (Fig. 6f). In addition, compared with the control and AdLC3, Adp53 significantly increased the apoptosis rate (Fig. 6g-k).

Altogether, Adp53 increased autophagy and apoptosis, which were accompanied by decreased collagen and Bcl-xL expression levels. The direct inhibition of Bcl-xL by sibcl$\mathrm{xL}$ resulted in decreased autophagy, increased apoptosis, and decreased collagen expression levels. Thus, wtp53induced autophgic apoptosis in the HSFs, which could explain the decreased collagen expression levels. However, sibcl-xL inhibited autophagy and thus decreased survival in the HSFs, ultimately resulting in apoptosis and reduced collagen expression levels. Altogether, both the excessive autophagy induced by wtp53 and the inhibition of autophagy by sibcl-xL led to decreased collagen expression levels, confirming the importance of maintaining autophagy balance.

\section{Adp53, AdLC3, shLC3, siBcl-2, and siBcl-xL reduce scar formation on rabbit ears}

Our in vitro study demonstrated that wtp53-modulated autophagic apoptosis inhibited HSF fibrosis. To further confirm the effect of autophagy on the continuous process of scar formation, cutaneous excision wound models were established using Netherland rabbits treated with i.d. injections of Adp53, shp53, AdLC3, shLC3, sibcl-2, and sibcl-xL. Convincingly, the results showed that the scar appearances in the Adp53, AdLC3, shLC3, sibcl-2, and sibcl-xL groups (Fig. 7a) were smaller and flatter than those in the shp53 and control groups (Fig. 7a), SEI (Fig. 7b, c) and the percentage of collagen fibers (Fig. S3a-c) were decreased 7 weeks after the treatment with Adp53, shLC3, and sibcl-xL. In addition, Masson's trichrome staining revealed that the collagen fibers in the rabbit ear scar model tissue (Fig. 8b) had a more disordered structure and were denser than those in the normal rabbit ear tissues (Fig. 8a); moreover, during the scar remodeling process, treatment with Adp53 (Fig. 8e), AdLC3 (Fig. 8f), shLC3 (Fig. 8i), sibcl-2 (Fig. 8k), and sibcl-xL (Fig. 81) resulted in a more neatly arranged and thinner structure than that observed in the control groups (Fig. 8b-d, j). In contrast, shp53 (Fig. 8h) led to a more disordered structure and denser collagen fibers than those observed in the control groups (Fig. 8g, j). The Adp53, shLC3, and sibcl-xL groups (Fig. 8e, i, l) were similar to the normal tissue (Fig. 8a). These results further confirm that wtp53-modulated HSF autophagy and autophagic HSF apoptosis regulated HSFs and inhibited HS formation in vivo.

\section{Discussion}

Hypertrophic scarring is a serious fibrotic skin disease that is esthetically displeasing and obstructs normal muscle function; thus, this disease causes psychological and physical suffering [1, 4-6]. Statistically, the incidence of HSs ranges from 40 to $70 \%$ following surgery and up to $91 \%$ following burn injury [46]. Currently, no effective therapeutically relevant targets have been reported for HS treatment, which is primarily due to the undefined molecular mechanism of HSs [47, 48]. Dermal fibroblasts are the most important effector cells responsible for HS formation [49]. Aberrant proliferation and apoptosis in fibroblasts, excessive ECM deposition, and $\alpha$-SMA overexpression often result in pathological scars [46-49]. Thus, inhibiting over proliferation, activating fibroblasts apoptosis, and reducing collagen-based ECM deposition are highly important for HS treatment.

Recent studies [16-20, 22-28] have shown that dysregulation of autophagy is associated with the pathogeneses 
$\mathbf{a}$

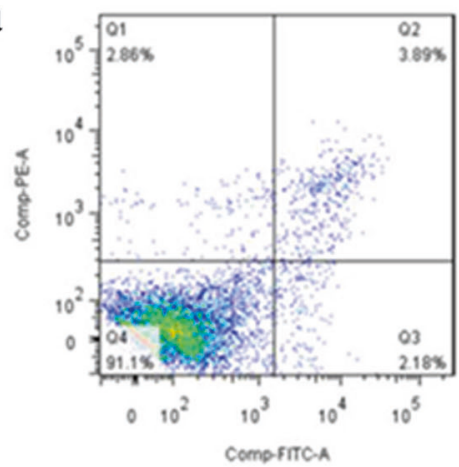

d

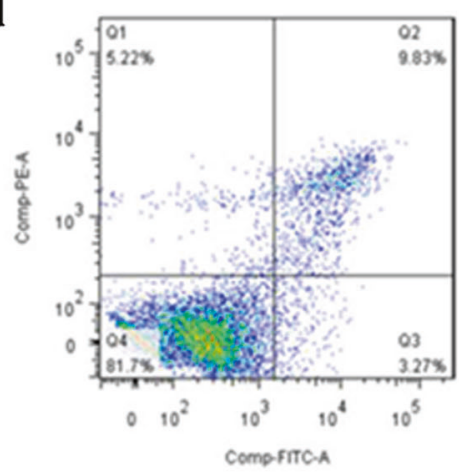

g

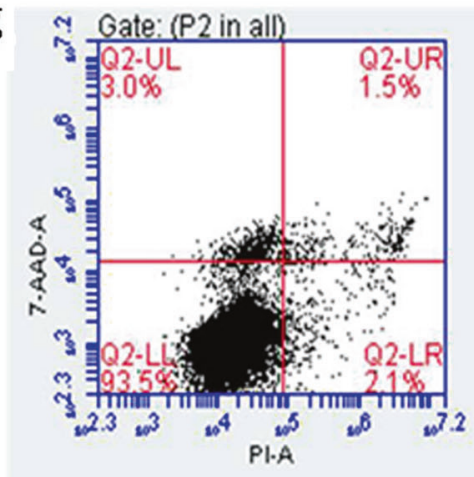

j

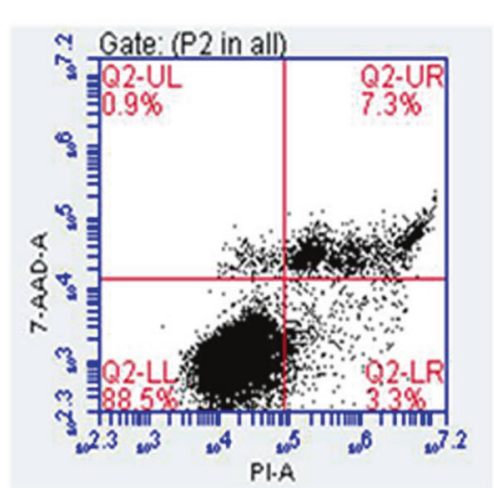

b

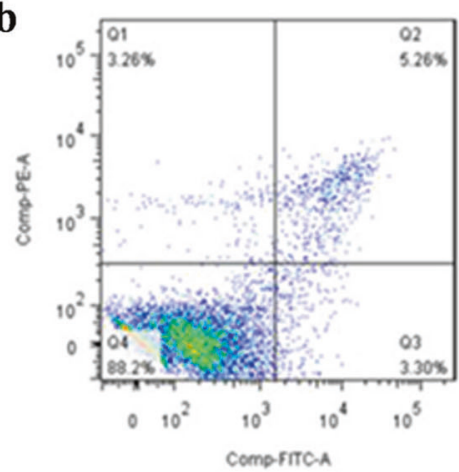

$\mathbf{e}$

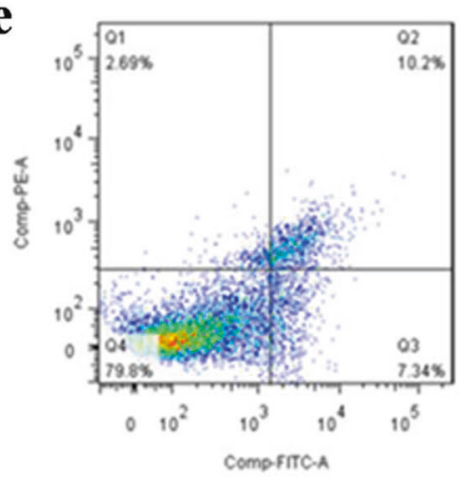

h

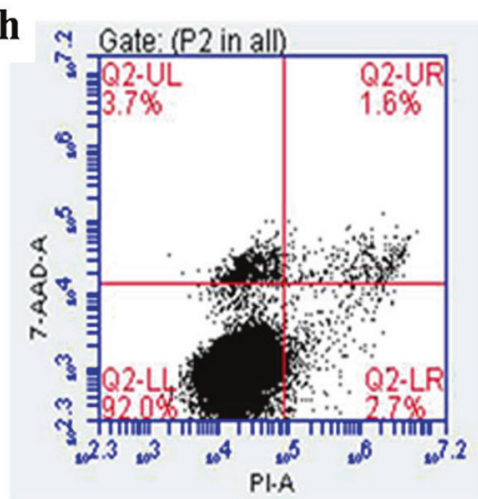

$\mathbf{k}$

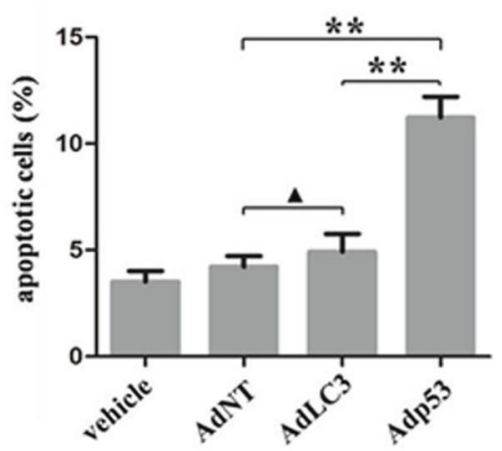

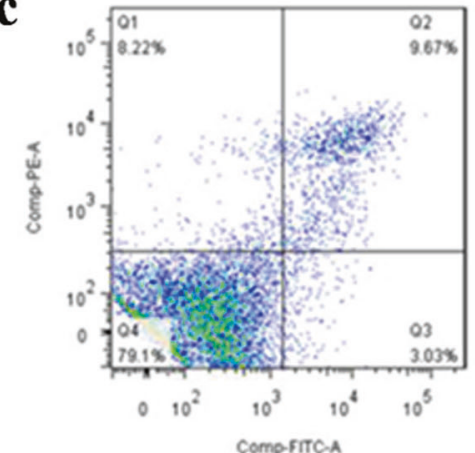

f

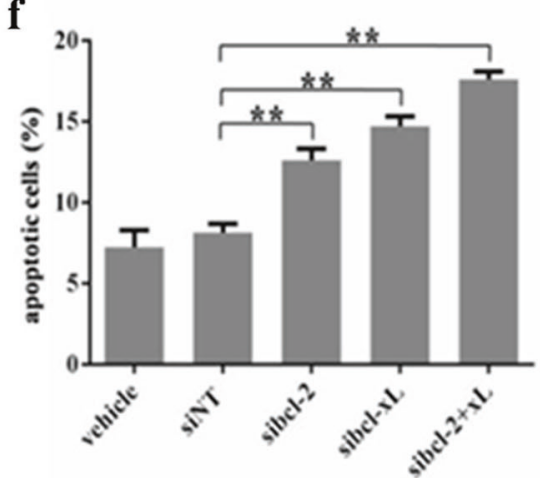

i

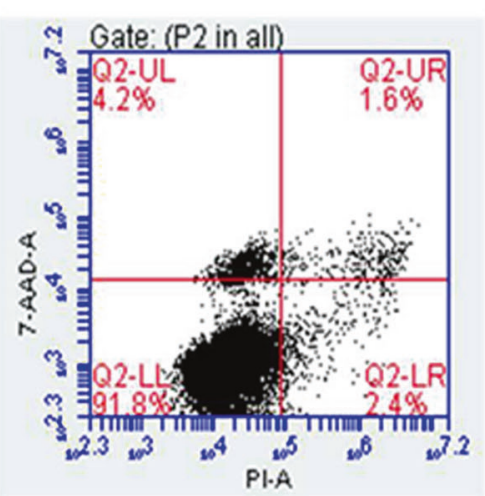

Fig. $6 \mathrm{Bcl}-\mathrm{xL}$ and wtp53 regulate fibroblast apoptosis. HSFs that were 70-80\% confluent were transfected with sibcl-2 (c, f), sibcl-xL (d, f), sibcl-2+xL (e, f), AdLC3 (i, k), Adp53 (j, k), and their negative controls $(\mathbf{a}, \mathbf{b}, \mathbf{g}, \mathbf{h}, \mathbf{f}, \mathbf{k})$ and cultured in DMEM for $48 \mathrm{~h}$.
Apoptosis was measured by flow cytometry using Annexin V and propidium iodide staining. Blank cells were used as controls. The data are representative of three experiments. $\mathrm{n}=3, \boldsymbol{\Delta}_{\mathrm{p}}>0.05, * \mathrm{p}<0.05$, $* * \mathrm{p}<0.01$ compared with the negative control group 


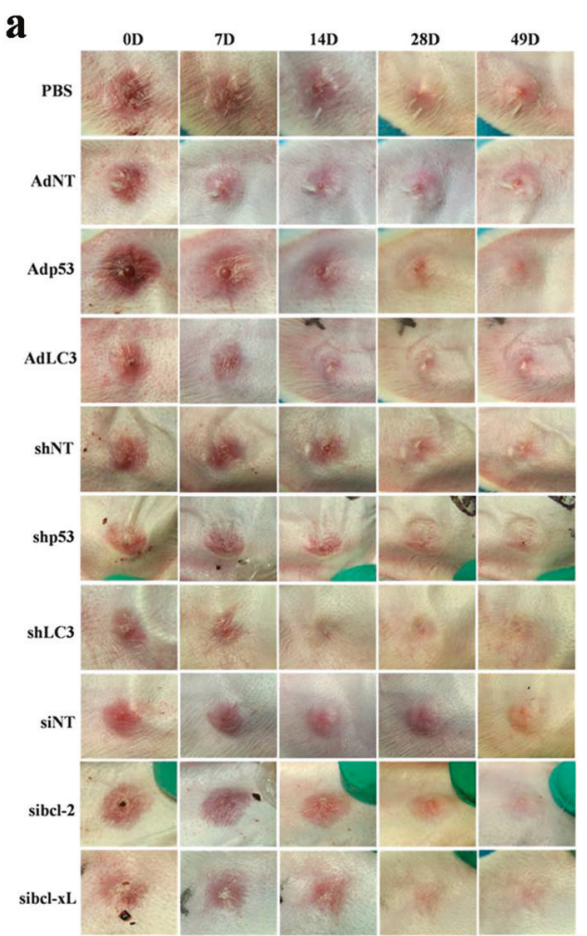

Fig. 7 Wtp53 overexpression, LC3 knockdown, and Bcl-xL silencing can improve the appearances of rabbit scar models. Rabbits were anesthetized, and four full-thickness wounds were created; then, the wounds were covered with sterile gauze. On postoperative day 28 , the scars were randomly allocated to the following ten groups: four control groups (PBS, AdNT, shNT, and siNT) and six treatment groups (Adp53, shp53, AdLC3, shLC3, sibcl-2, and sibcl-xL). a After

of many diseases in mammals. Our previous studies [6, 35] show that IL-10 has potential therapeutic benefits of preventing and reducing HS formation primarily because of the IL-10-mediated autophagy inhibition in HSFs. Previous studies $[17,50,51]$ suggest that autophagy in the skin dermis is associated with the maintenance, viability, differentiation, and survival of fibroblasts in wound healing and leads to the pathogenesis of pathological scars. We further found that the autophagic capacity in HSs/HSFs is higher than that in NS/NSFs (Fig. S1). However, the specific molecule and the mechanism by which it regulates HSF autophagy must be further elucidated.

p53 is a transcription factor that performs a central function in the regulation of autophagy and apoptosis [30, 31, 52]. Currently, whether p53 regulates autophagy in positive or negative ways remains controversial. In addition, a previous study [53] found that 18 of 20 keloids overexpressed p53 at the protein level, while other studies [54] have reported that p53 was not overexpressed in keloids or HS. Therefore, it is necessary to confirm the effect of p53-modulated autophagy and autophagic HSF apoptosis on HS fibrosis. Our result showed that wtp53 promoted HSF autophagy and autophagic apoptosis and that collagen expression was inhibited. b

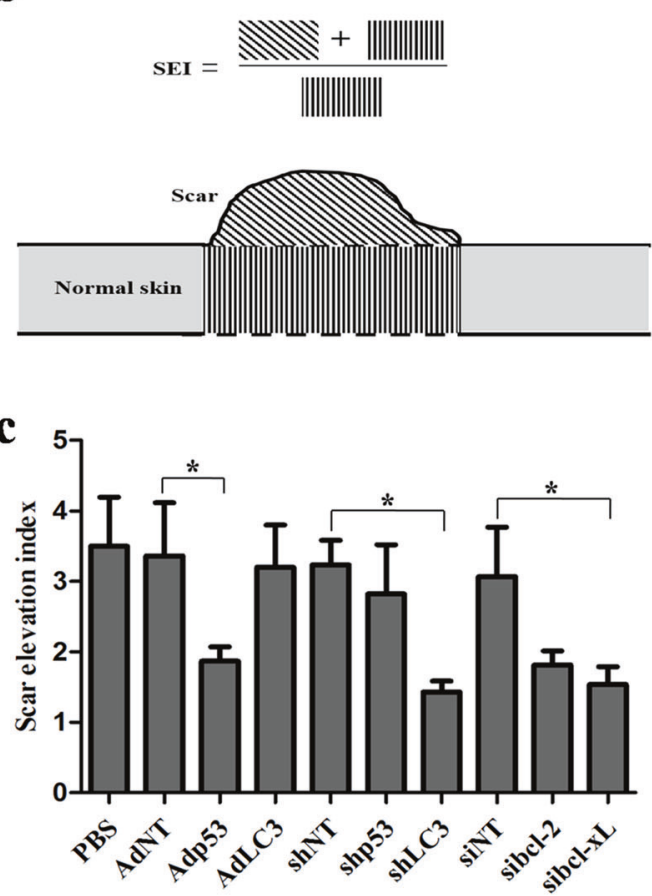

treatment for $7,14,28$, and 49 days, the scar appearance analyses show that the scars in the Adp53 and shLC3 groups were smaller and flatter than those in the shp53 and AdLC3 groups. b The schematic diagram of scar elevation index (SEI), it is reflected the degree of scar hypertrophy. c After treatment with Adp53, shLC3, and sibcl-xL for 49 days, SEI decreased. The data are representative of six experiments. $n=6, * p<0.05$ compared with the control group

$\mathrm{Bcl}-\mathrm{xL}$, which is an antiapoptotic member of the Bcl-2 family of proteins, acts as a pro-survival protein by preventing the release of mitochondrial contents and interfering with programmed cell death independently of promoting cell division [55-57]. Many studies [58] have reported that Bcl-2 is a prototypical inhibitor of apoptosis (type I programmed cell death) and that Bcl-2 regulates autophagy in pancreatic cancer [59]. Our results showed that sibcl-xL, but not sibcl-2, downregulated the expression of fibrotic factors (Figs. 3 and 5) and HSF autophagy (Fig. 5), whereas Adp53 upregulated autophagy and inhibited collagen, which appears contradictory.

To investigate this discrepancy, flow cytometry was performed and showed that sibcl-2 and sibcl-xL significantly increased HSF apoptosis (Fig.6). In addition, only sibcl-xL inhibited autophagy and the expression of fibrotic factors in HSFs. Wtp53 also increased autophagic apoptosis in HSFs. Based on these findings, wtp53 modulated the inhibition of HSF fibrosis by disrupting the balance of autophagy and leading to autophagic HSF apoptosis, whereas sibcl-xL inhibited fibrosis by inhibiting autophagy and survival in HSFs.

By combining gene overexpression, knockdown, and silencing technologies, our experiments revealed that 


\section{a}

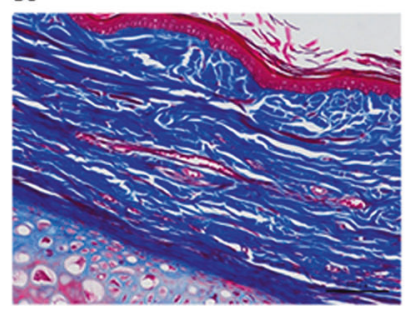

d

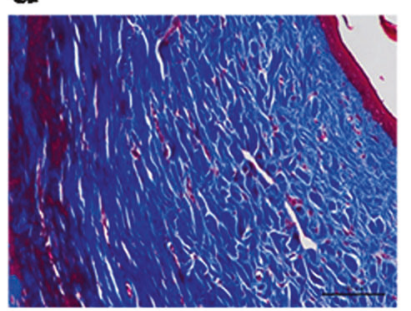

g

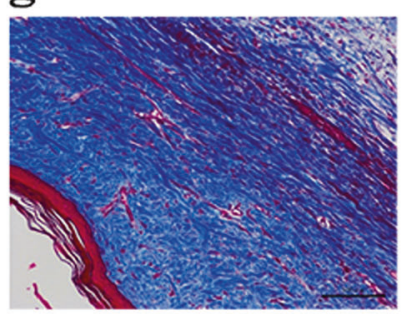

j

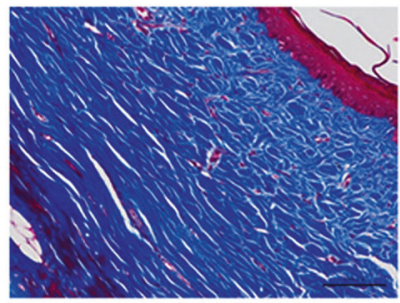

b

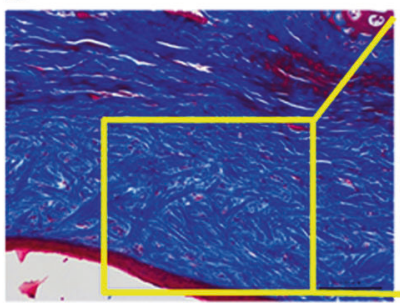

e

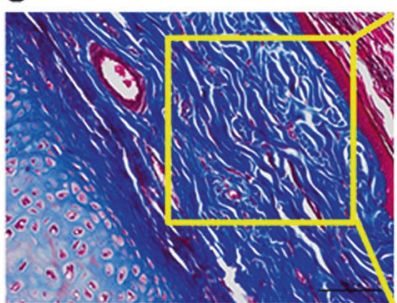

h

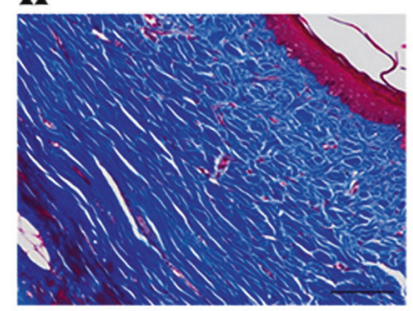

$\mathbf{k}$

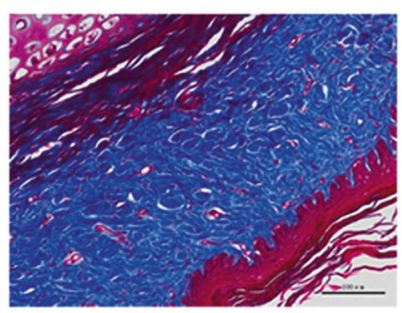

Fig. 8 Wtp53 overexpression, LC3 knockdown, and Bcl-xL silencing can reduce the deposition and improve the arrangement of collagen fibers in rabbit scar models. Masson's trichrome staining of collagen fibers in the rabbit scar models. a Normal rabbit ear skin with regularly arranged, small collagen fibers. b Rabbit ear scar model tissue with thicker, denser, and disorganized collage fibers. $\mathbf{c}, \mathbf{d}, \mathbf{f}, \mathbf{g}, \mathbf{h}, \mathbf{j}, \mathbf{k}$ In the PBS, AdNT, AdLC3, shNT, shp53, siNT, and sibcl-2 groups,

Adp53, shLC3, and sibcl-xL downregulated the expression levels of both Col 1 and $\mathrm{Col} 3$, reduced ECM accumulation (Figs. 3 and 5), and altered collagen arrangement (Fig. 8). More persuasively, the rabbit ear scar models injected with Adp53, AdLC3, shLC3, sibcl-2, or sibcl-xL yielded a smaller and flatter scar appearance (Fig. 7a), and theses scars displayed a more neatly arranged, thinner structure (Fig. 7a), lower SEI (Fig. 7b, c) and lower the percentage of collagen fibers (Fig. S3) than those in the control groups (Fig. 7a, c). It appears to be contradictory that AdLC3 could improve the appearance of the rabbit ear scars but not

c
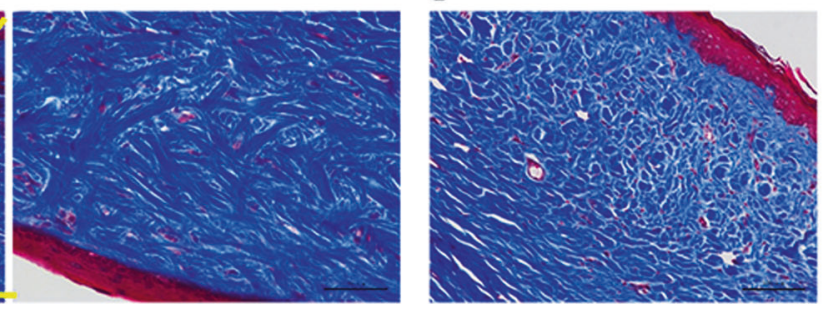

f
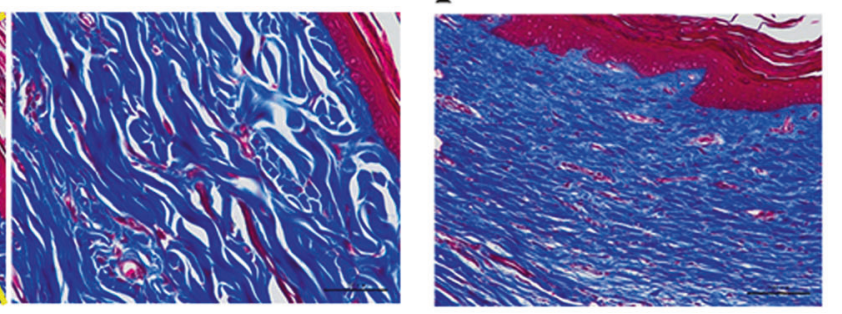

i

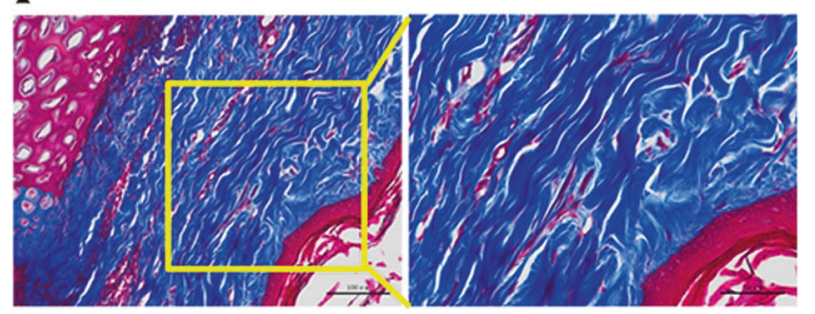

I
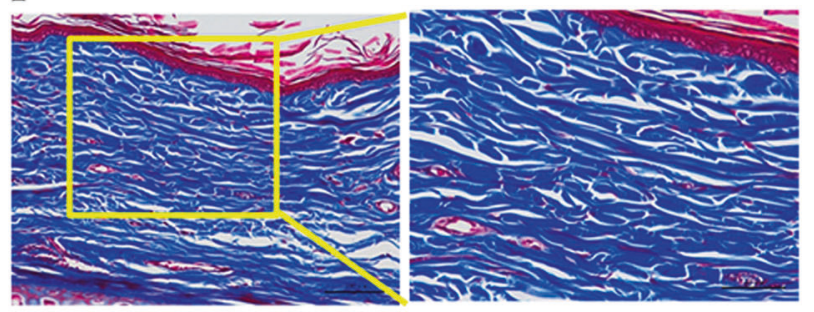

the amount of collagen fibers was much greater than that in the normal group, and the collage fibers were thicker, denser, and disorganized. e, i, l After treatment with Adp53, shLC3, and sibcl-xL for 49 days, the collagen fibers were more regularly arranged and sparser than those in the model, PBS and corresponding control groups. $n=$ 6 scars per group. Scale bars, 100 and $50 \mu \mathrm{m}$

suppress the expression of Col 1 and Col 3 in the HSFs; these results might have occurred because AdLC3 primarily changed the architecture arrangement of $\alpha$-SMA, further affecting its function in HSFs. Therefore, the balance of autophagy in HSFs is critical for fibrosis formation, and both the downregulate and excessive upregulation of autophagy could inhibit collagen expression. In addition, regulating HSF autophagy and autophagic HSF apoptosis by targeting p53 and Bcl-xL may be an effective method for treating HS and related fibrotic diseases. The mechanism involved in the process must be further studied. 
In conclusion, we identified that wtp53 inhibited HS fibrosis by inducting autophagy and autophagic HSF apoptosis in vitro (HSFs) and in vivo (rabbit ear scar model). Wtp53-modulated autophagy and autophagic HSF apoptosis might serve as potential molecular targets for HS therapy.

Acknowledgements This study was supported by the National Natural Science Foundation of China (Grant Nos. 81571914 and 81741105) and the Natural Science Foundation of Shaanxi Province of China (No. 2015JM8452).

\section{Compliance with ethical standards}

Conflict of interest The authors declare that they have no conflict of interest.

\section{References}

1. Aarabi S, Longaker MT, Gurtner GC. Hypertrophic scar formation following burns and trauma: new approaches to treatment. PLoS Med. 2007;4:e234.

2. Akkoç Y, Berrak Ö, Arısan ED, et al. Inhibition of PI3K signaling triggered apoptotic potential of curcumin which is hindered by Bcl-2 through activation of autophagy in MCF-7 cells. Biomed Pharmacother. 2015;71:161-71.

3. Armour A, Scott PG, Tredget EE. Cellular and molecular pathology of HTS: basis for treatment. Wound Repair Regen. 2007;15((Suppl.1):S6-17.

4. Bayat A, McGrouther DA, Ferguson MW. Skin scarring. BMJ. 2003;326:88-92.

5. Brown BC, McKenna SP, Siddhi K, et al. The hidden cost of skin scars: quality of life after skin scarring. J Plast Reconst Aesthet Surg. 2008;61:1049-58.

6. Shi JH, Guan H, Shi S, et al. Protection against TGF- $\beta 1$-induced fibrosis effects of IL10 on dermal fibroblasts and its potential therapeutics for the reduction of skin scarring. Arch Dermatol Res. 2013;305:341-52.

7. Sidgwick GP, Bayat A. Extracellular matrix molecules implicated in hypertrophic and keloid scarring. J Eur Acad Dermatol Venereol. 2012;26:141-52.

8. van der Veer WM, Bloemen MC, Ulrich MM, et al. Potential cellular and molecular causes of hypertrophic scar. Burns. 2009;35:15-29.

9. Beanes SR, Dang C, Soo C, et al. Skin repair and scar formation: the central role of TGF-beta. Expert Rev Mol Med. 2003;5:1-22.

10. Campaner AB, Ferreira LM, Gragnani A, et al. Upregulation of TGF-betal expression may be necessary but is not sufficient for excessive scarring. J Invest Dermatol. 2006;126:1168-76.

11. Liu W, Wang DR, Cao YL. TGF-beta: a fibrotic factor in wound scarring and a potential target for anti-scarring gene therapy. Curr Gene Ther. 2004;4:123-36.

12. Sarrazy V, Billet F, Micallef L, et al. Mechanisms of pathological scarring: role of myofibroblasts and current developments. Wound Repair Regen. 2011;19(Supp11):s10-5.

13. Hara T, Nakamura $K$, Matsui $M$, et al. Suppression of basal autophagy in neural cells causes neurodegenerative disease in mice. Nature. 2006;441:885-9.

14. Kondo Y, Kanzawa T, Sawaya R, et al. The role of autophagy in cancer development and response to therapy. Nat Rev Cancer. 2005;5:726-34

15. Bhoopathi P, Chetty C, Gujrati M, et al. Cathepsin B facilitates autophagy-mediated apoptosis in SPARC overexpressed primitive neuroectodermal tumor cells. Cell Death Differ. 2010;17: 1529-39.

16. Portt L, Norman G, Clapp C, et al. Anti-apoptosis and cell survival: a review. Biochim Biophys Acta. 2011;1813:238-59.

17. Levine B, Kroemer G. Autophagy in the pathogenesis of disease. Cell. 2008;132:27-42.

18. Meijer AJ, Dubbelhuis PF. Amino acid signaling and the integration of metabolism. Biochem Biophys Res Commun. 2004;313:397-403.

19. Riddle DL, Gorski SM. Shaping and stretching life by autophagy. Dev Cell. 2003;5:364-5.

20. Yoshimori T. Autophagy: a regulated bulk degradation process inside cells. Biochem Biophys Res Commun. 2004;313:453-8.

21. HouW HanJ, Lu C, et al. Autophagic degradation of active caspase-8: a crosstalk mechanism between autophagy and apoptosis. Autophagy. 2010;6:891-900.

22. Jin S, White E. Tumor suppression by autophagy through the management of metabolic stress. Autophagy. 2008;4:563-6.

23. Orvadahl A, Levine B. Eating the enemy within: autophagy in infectious diseases. Cell Death Differ. 2009;16:57-69.

24. Shi JH, Hu DH, Zhang ZF, et al. Reduced expression of microtubule-associated protein 1 light chain 3 in hypertrophic scars. Arch Dematol Res. 2012;304:209-15.

25. Shintani T, Klionsky DJ. Autophagy in heath and disease: a double-edged sword. Science. 2004;306:990-5.

26. Todde V, Veenhuis M, van der Klei IJ. Autophagy: principles and significance in health and disease. Biochim Biophy Acta. 2009;1792:3-13.

27. Winslow AR, Rubinsztein DC. Autophagy in neurodegeneration and development. Biochim Biophys Acta. 2008;1782:723-9.

28. Yen WL, Klionsky DJ. How to live long and prosper: autophagy, mitochondria, and aging. Physiology. 2008;23:248-62.

29. De Felice B, Garbi C, Santoriello M, et al. Differential apoptosis markers in human keloids and hypertrophic scars fibroblasts. Mol Cell Biochem. 2009;327:191-201.

30. Duan L, PerezR E, Davaadelger B, et al. p53-regulated autophagy is controlled by glycolysis and determines cell fate. Oncotarget. 2015;6:23135-56.

31. Seillier M, Peuget S, Gayet O, et al. TP53INP1, a tumor suppressor, interacts with LC3 and ATG8-family proteins through the LC3-interacting region (LIR) and promotes autophagy-dependent cell death. Cell Death Differ. 2012;19:1525-35.

32. Ikeda K, Torigoe T, Matsumoto $\mathrm{Y}$, et al. Resveratrol inhibits fibrogenesis and induces apoptosis in keloid fibroblasts. Wonud Repair Regen. 2013;21:616-23.

33. Djafarzadeh R, Notohamiprodjo S, Rieth N, et al. Treatment of dermal fibroblasts with GPI-anchored human TIMP-1 protein moderates processes linked toscar formation. J Invest Dermatol. 2013;133:803-11.

34. Akasaka Y, Ono I, Tominaga A, et al. Basic fibroblast growth factor in an artificial dermis promotes apoptosis and inhibits expression of alpha-smooth muscle actin, leading to reduction of wound contraction. Wound Repair Regen. 2007;15:378-89.

35. Shi J, Wang $\mathrm{H}$, Guan $\mathrm{H}$, et al. IL10 inhibits starvation-induced autophagy in hypertrophic scar fibroblasts via cross talk between the IL10-IL10R-STAT3 and IL10-AKT-mTOR pathways. Cell Death Dis. 2016;7:e2133.

36. Hu X, Wang H, Liu J, et al. The role of ERK and JNK signaling in connective tissue growth factor induced extracellular matrix protein production and scar formation. Arch Dermatol Res. 2013;305:433-45.

37. $\mathrm{Hu} \mathrm{X}, \mathrm{Li} \mathrm{N}$, Tao K, et al. Effects of integrin $\alpha \nu \beta 3$ on differentiation and collagen synthesis induced by connective tissue growth factor in human hypertrophic scar fibroblasts. Int J Mol Med. 2014;4:1323-34. 
38. Bai X, Fan L, He T, et al. SIRT1 protects rat lung tissue against severe burn-induced remote ALI by attenuating the apoptosis of PMVECs via p38 MAPK signaling. Sci Rep. 2015;5:10277.

39. Bai X, He T, Liu J, et al. Loureirin B inhibits fibroblast proliferation and extracellular matrix deposition in hypertrophic scar via TGF- $\beta$ /Smad pathway. Exp Dermatol. 2015;24:355-60.

40. Zhao B, Liu JQ, Yang C, et al. Human amniotic epithelial cells attenuate TGF- $\beta 1$-induced human dermal fibroblast transformation to myofibroblasts via TGF- $\beta 1 / \mathrm{Smad} 3$ pathway. Cytotherapy. 2016;18:1012-24.

41. Marchenko ND, Moll UM. Mitochondrial death functions of p53. Mol Cell Oncol. 2014;1:e955995.

42. Xu-Monette ZY, Medeiros LJ, Li Y, et al. Dysfunction of the TP53 tumor suppressor gene in lymphoid malignancies. Blood. 2012;119:3668-83.

43. Burton TR, Gibson SB. The role of Bcl-2 member BNIP3 in cell death and disease: NIPping at the heels of cell death. Cell Death Differ. 2009;16:515-23.

44. Gabriel B, Sureau F, Casselyn M, et al. Retroactive pathway involving mitochondria in electroloaded cytochrome c-induced apoptosis. Protective properties of Bcl-2 and Bcl-XL. Exp Cell Res. 2003;289:195-210

45. Ko JK, Choi KH, Peng J, et al. Amphipathic tail-anchoring peptide and $\mathrm{Bcl}-2$ homology domain-3 (BH3) peptides from $\mathrm{Bcl}-2$ family proteins induce apoptosis through different mechanisms. $\mathrm{J}$ Biol Chem. 2011;286:9038-48.

46. Honardoust D, Ding J, Varkey M, et al. Deep dermal fibroblasts refractory to migration and decorin-induced apoptosis contribute to hypertrophic scarring. J Burn Care Res. 2012;33:668-77.

47. Saito M, Yamazaki M, Maeda T, et al. Pirfenidone suppresses keloid fibroblast-embedded collagen gel contraction. Arch Dermatol Res. 2012;304:217-22.
48. Gauglitz GG, Korting HC, Pavicic T, et al. Hypertrophic scarring and keloids: pathomechanisms and current and emerging treatment strategies. Mol Med. 2011;17:113-25.

49. Hinz B. Formation and function of the myofibroblast during tissue repair. J Invest Demmatol. 2007;127:526-37.

50. Lock R, Debnath J. Extracellular matrix regulation of autophagy. Curr Opin Cell Biol. 2008;20:583-8.

51. Vellai T, Bicsák B, Tóth ML, et al. Regulation of cell growth by autophagy. Autophagy. 2008;4:507-9.

52. Woods DB, Vousden KH. Regulation of p53 function. Exp Cell Res. 2001;264:54-66.

53. Ladin DA, Hou Z, Patel D, et al. p53 and apoptosis alterations in keloids and keloid fibroblasts. Wound Repair Regen. 1998;6:28-37.

54. Teofoli P, Barduagni S, Ribuffo M, et al. Expression of Bcl-2, p53, c-jun and c-fos protooncogenes in keloids and hypertrophic scars. J Dermatol Sci. 1999;22:31-7.

55. Saxena N, Katiyar SP, Liu Y, et al. Molecular interactions of Bcl2 and Bcl-xL with mortalin: identification and functional characterization. Biosci Rep. 2013;33:e0073.

56. Petros AM, Olejniczak ET, Fesik SW. Structural biology of the Bcl-2 family of proteins. Biochim Biophys Acta. 2004;1644:83-94.

57. Thomadaki H, Scorilas A. Bcl-2 family of apoptosis-related genes: functions and clinical implications in cancer. Crit Rev Clin Lab Sci. 2006;43:1-67.

58. Qian J, Voorbach MJ, Huth JR, et al. Discovery of novel inhibitors of Bcl-xL using multiple high-throughput screening platforms. Anal Biochem. 2004;328:131-8.

59. Hari Y, Harashima N, Tajima $\mathrm{Y}$, et al. Bcl-xL inhibition by molecular-targeting drugs sensitizes human pancreatic cancer cells to TRAIL. Oncotarget. 2015;6:41902-15. 